\title{
Study of the effect of seismically-induced geoelectric and geomagnetic fields on secondary particle detection at a LAGO site.
}

\author{
Diego Alberto Coloma Borja ${ }^{a, *}$ and Edgar Carrera Jarrin ${ }^{a}$ on behalf of the \\ LAGO Collaboration \\ (a complete list of authors can be found at the end of the proceedings) \\ ${ }^{a}$ Universidad San Francisco de Quito, \\ Diego de Robles s/n, Quito, Ecuador \\ E-mail: dacolomab@alumni.usfq.edu.ec, ecarrera@usfq.edu.ec
}

The LAGO (Latin American Giant Observatory) project is an extended Astroparticle Observatory at global scale. It is mainly oriented to basic research on three branches of Astroparticle physics: the Extreme Universe, Space Weather phenomena, and Atmospheric Radiation at ground level. This work is framed in the latter branch, its aim is to study the potential effects on extensive air showers from changes in geoelectric and geomagnetic fields, induced by the onset of seismic activity. For this purpose, simulations for flux of secondaries are performed with ARTI, a tool developed by LAGO that combines Magnetocosmics, CORSIKA, and Geant4 to account, respectively, for the propagation of a shower by a primary particle, geomagnetic corrections, and detector response. Using ARTI, we have calculated the background radiation at the LAGO Site in Universidad San Francisco de Quito (2200 m.a.s.l). Regular conditions for the Earth's electromagnetic field are taken from the NCEI Geomagnetic Calculator for specific fair-weathered days above the location. Variations from this regularity are introduced based on relevant studies on seismic activity. The results show that there exists an effect on the number of secondary particles at ground level, which could, in principle, be detected by a LAGO WCD detector.

$3^{\text {th }}$ International Cosmic Ray Conference (ICRC 2021)

July 12 th - 23rd, 2021

Online - Berlin, Germany

\footnotetext{
${ }^{*}$ Presenter
} 


\section{Introduction}

The Latin America Giant Observatory (LAGO) project is an Astroparticle Observatory built extensively by a network of institutions throughout nine Latin American countries: Argentina, Bolivia, Brazil, Colombia, Chile, Ecuador, Guatemala, Mexico, Peru and Spain [1]. One of its main scientific objectives is to study the effects of atmospheric phenomena as relevant to the entry of cosmic rays and subsequent particle detection at ground level. This is accomplished through the work at the different sites that constitute the LAGO detection. Each of this LAGO sites consists of one or multiple Water Cherenkov Detectors (WCD) at distinct altitudes, and geomagnetic rigidity cut-offs.

Universidad San Francisco de Quito is one of three Ecuadorian institutions part of the LAGO Collaboration. There is a planned site to install a WCD within the institution, located at 2200 m.a.s.1 . Bearing in mind that the Andes range in Ecuador is a region of high seismic activity, we understand that placing a detector at this site could represent a great opportunity to study the possibility of finding precursor effects of earthquakes on particle detection in a WCD.

Currently, the mechanisms at play within seismic activity are not fully understood. However, some studies have shown a relationship between the onset of seismic activity and changes in the measurement of local geoelectric and geomagnetic fields [2-8]. Among this, a study performed in 2018 observed and studied geomagnetic changes in relation to different seismic events for which records exist[9]. This study finds different levels of responses to the onset of earthquakes. From the data taken, and in relevance to this study, we take away an upper limit for change in geomagnetic field $\Delta B_{z} \approx 0.20 \mu \mathrm{T}$. Moreover, a 2013 study analyzed the incidence of vertical electric fields as precursory events to the onset of seismic activity in fair-weather days [10]. This study found changes between $-0.457 \mathrm{kV} / \mathrm{m}$ and $-1.38 \mathrm{kV} / \mathrm{m}$ as precursors for seismic events of magnitudes between 4.3 and 7.8.

For this study, we use the CORSIKA 7.5600 code[11] as established within LAGO to simulate secondary particle arrival at the USFQ site, introducing changes in vertical geoelectric and geomagnetic field parameters and comparing them to a baseline established to be regular conditions. In first place, we perform simulations for the secondary arrival produced by a single $10^{4} \mathrm{GeV}$ proton entering the atmosphere vertically above the site of detection. Then, we perform similar simulations for one hour of particle flux prepared using the ARTI package $[12,13]$ for simulation of flux of primaries. Geomagnetic parameters for regular conditions are estimated from a magnetic field calculator[14] for a fair-weather day above the city of Quito. Additionally, baseline for electric fields are taken to be zero assuming no other relevant events that could affect the geoelectric field for said day. For both the single proton and one hour set of simulations, we take into account these baseline set of parameters for the regular or typical conditions simulations. Next, we perform two additional simulations in each case changing the magnetic and electric field parameters respectively with respect to the baseline.

\section{Simulation Parameters}

For the simulation, baseline parameters are taken from conditions above the city of Quito on November 13, 2020: $B x$ 11.031 $\mu \mathrm{T}, B z 4.081 \mu \mathrm{T}$. Simulations for a single proton are averaged 
from ten similar simulations, each with different seeds. On the other hand, for one hour flux, we take the conditions from November 13, 2020: For this set of simulations we set the zenith angles of entry between $0^{\circ}$ and $90^{\circ}$, and azimuth angles between -180 and $180^{\circ}$. As well as primary particle energies between: $\left(5 \mathrm{GeV}, 10^{6} \mathrm{GeV}\right)$.

Due to limited availability of data for electromagnetic shifts related to seismic activity, the values for the change in parameters are taken from different studies. For the change in geomagnetic field, we took a variation of $\Delta B_{z} \quad 0.25 \mu \mathrm{T}$, since this is a value close to the upper limit as presented in [9]. On the other hand, for the geoelectric variation, we took $\Delta E_{z} 10 \mathrm{Vcm}$, which is a central value for the results found in certain seismic events that could be oberved in [10].

\section{Simulation Results}

\subsection{Showers initiated by a single proton}

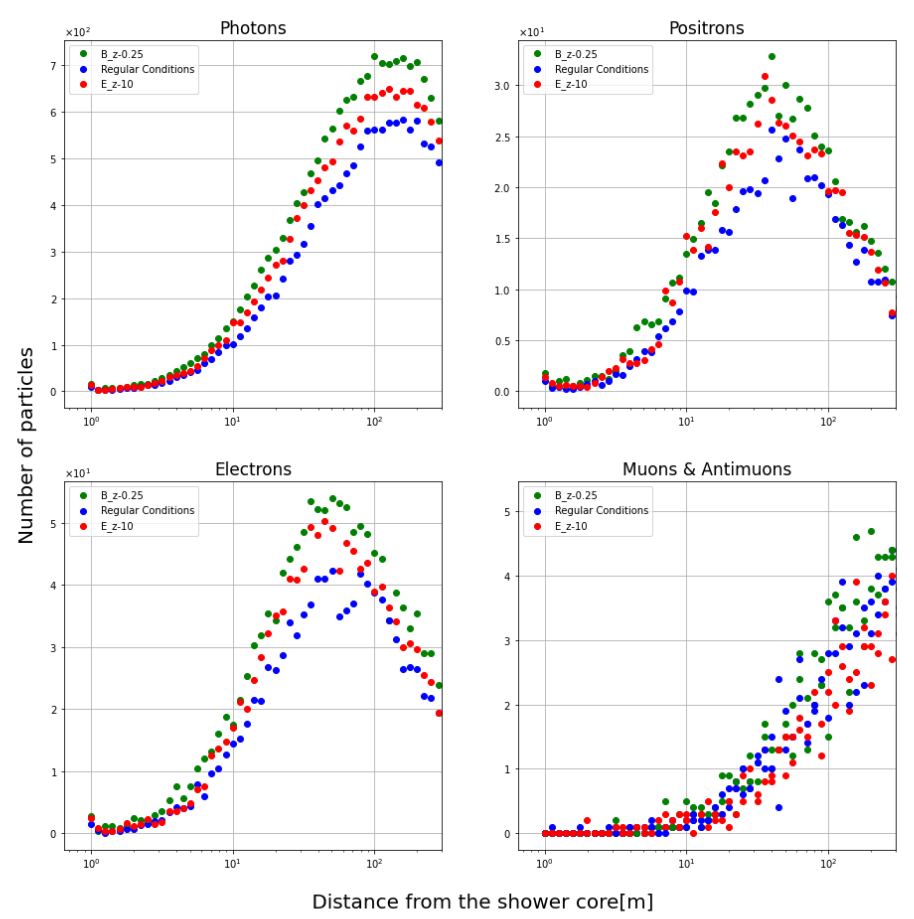

Figure 1: Distribution of secondary particle number at ground level with respect to absolute distance from the detector position.the electromagnetic and muonic components from an air shower from a $10^{4} \mathrm{GeV}$ proton. Some differences can be observed between distributions

Results of simulations using protons proton as cosmic ray primary particles can be seen in Fig. 1. All of the distributions for different particle types show behaviors expected for lateral particle distributions at ground level. Changes in the electric and magnetic field seem to show some difference in the distribution when compare to regular conditions for photons, electrons and positrons. For muons and antimuons, also pictured, there is no clear effect observed in this figure. 
Nevertheless, in order to properly analyze the results, it is pertinent to look at the relative difference of the distributions from regular conditions. This relative difference is calculated at each step of distance as: $\frac{N_{\text {change }}-N_{\text {base }}}{N_{\text {base }}}$. The distributions for this difference are pictured in Fig 2. This figure shows how much the distributions for changes in electromagnetic fields vary with respect to the base of regular conditions. To some extent, a certain degree of divergence can be attributed to the stochastic nature of particle simulations. However, there appears to be some degree of consistent shift when looking especially at photons. Of note is the fact that there is a spike in the percentage of difference closest to the origin of the distribution for photons, electrons and positrons. For muons and antimuons on the other hand, there is no consistent shift observed.

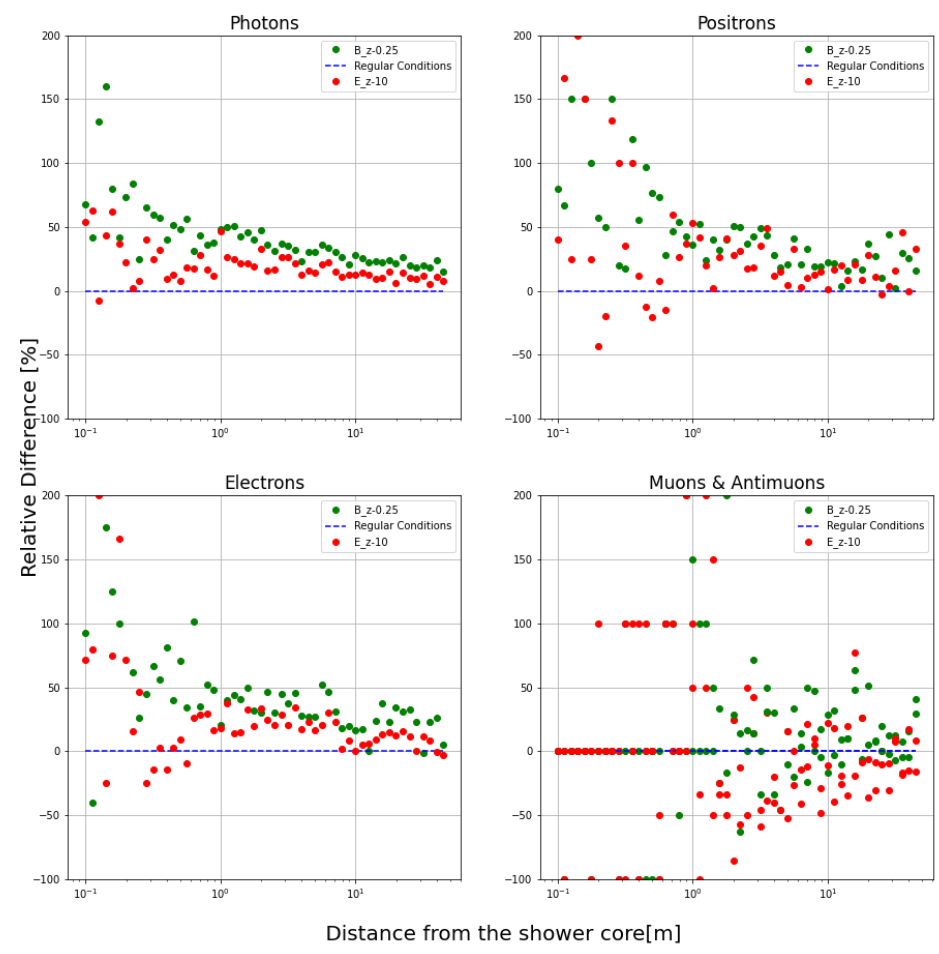

Figure 2: Relative difference of distributions of secondary particles at ground level from regular conditions for showers initiated by a single proton. There is some degree of consistency when looking at the changes in distribution for particles in the electromagnetic component of the shower, Which is most clear for photons. Photons, Electrons and Positron all show high changes at distances closer to the detector Changes in the muonic component appear to lack this consistency and could be attributed to randomness

Although the results of these simulations show a difference in distributions for changes in electromagnetic parameters, they are not enough to ascertain an effect of seismically-induced fields on simulated particle detection. To take into account a more realistic scenario, we have performed simulations of one hour flux of primaries as previously described in the introduction. 


\subsection{Showers created by a one hour flux of particle entry}

The following results stem from simulations performed for a set of showers corresponding to one hour of flux (3600 s) of cosmic rays. In this case, simulations are created from identical parameters except for the changes in geolectric and geomagnetic field discussed before. Which are performed in a similar fashion to the previous section.

Of note is the fact that this particular set of simulations, due to the high computational requirement, was performed taking advantage of the Google Cloud platform. Using Kubernetes cluster technologies established within the Cloud framework and the argo workflow manager, in addition to a set of Docker containers previously prepared with the simulations to be ran.

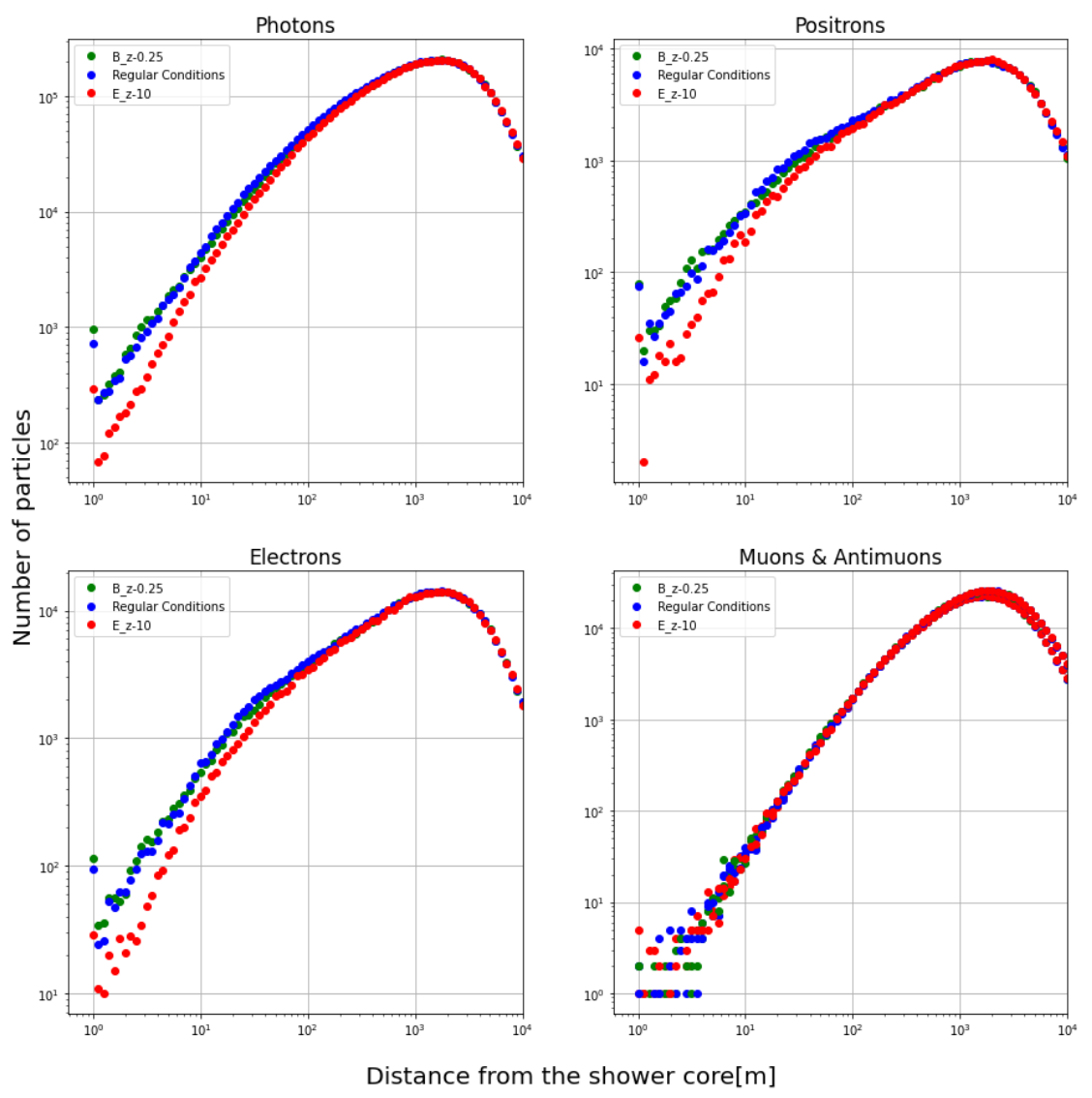

Figure 3: Distribution of secondary particle (photons, positrons, electrons \& muons) number at ground level with respect to absolute distance and in the vicinity of the center of coordinates. There is some difference observed between the distributions observed.

Results for one hour of flux simulations are shown in Fig.(3). The results do not show a clear difference between the distributions when changing geoelectric and geomagnetic parameters for the electromagnetic or muonic components. In order to draw more appropriate conclusions, Fig. 4 shows the percentage relative difference from regular conditions for both conditions of change. The picture shows that further from the detector, the distributions are close enough from one another to attribute deviations to the random process of simulation. Nevertheless, at closer distances to the shower core, the distribution presents a more significant change in comparison to the regular 
distribution. This is consistent with the fact that there is a smaller number of particles closer to the origin position and any deviations will therefore much more significant.

The results of a change in the magnetic field for photons, positrons and electrons present a difference in the distribution, increasing the number of particles with respect to the base up to $10^{0}$ $[\mathrm{m}]$, then presenting a decrease in particle number before settling back to the baseline. For the same particles, the distributions for change in electric field present a drop when compared to the baseline starting at the origin and up to $10^{1}[\mathrm{~m}]$. There seems to be a noticeable change on the other tail of the distribution for both cases. However, it doesn't appear to show a consistent form of change and could be attributed to randomness. For muons and their antiparticles, there seems to be a deviation from regular conditions. However, this deviation doesn't show a form of consistent increase or decrease in any area of the distribution.

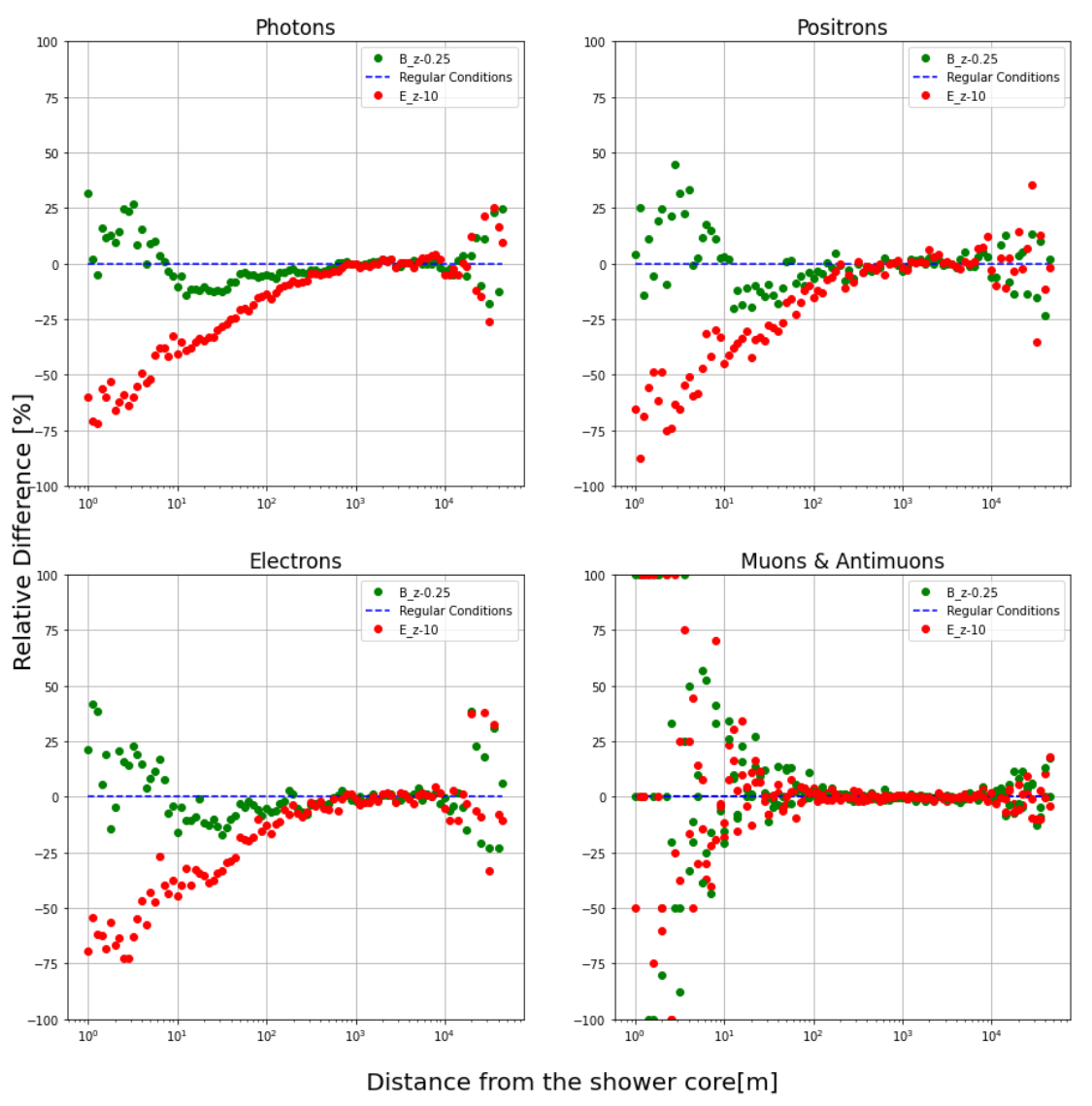

Figure 4: Relative difference of distributions of secondary particles at ground level from regular conditions for one-hour flux of particles. At distances close to the detector position, there is consistency when looking at the changes in distribution for particles in the electromagnetic component of the shower. The magnetic and electric field change distributions reproduce different but consistent forms of change for photons, positrons and electrons. Changes in the muonic component appear to lack this consistency and could be attributed to randomness 


\section{Conclusions}

The results presented show that the models and codes being employed for simulation of EAS within the LAGO collaboration - specifically CORSIKA within the ARTI package - reflect a certain variation of the electromagnetic particle distribution at ground level in response to changes in the value value of geoelectric and geomagnetic field parameters.

Simulations for one hour of particle flux for photons, electrons and positrons show a change in distribution in the cases of change in electric and magnetic fields. The form of the change of distribution for the magnetic field shows consistent increase and decrease in different areas of the simulation, reflecting a difference purely in the form of the distribution when a change in magnetic field is applied in the parameters of the simulation. Furthermore, this is consistent with the expectation that a change in vertical magnetic field might affect particle detection by affecting the trajectory of charged particles traveling downwards towards the Earth's surface

On the other hand, for the change in electric field, there is up to a 75\% drop in simulated particle arrival close to the shower core. This suggests a difference in the number of particles arriving at detector level in addition to a change in the distribution. A particle traveling through an electric field as simulated in CORSIKA will experiment acceleration, deceleration or deflection. Taking this into account, the main effect of the incidence of a vertical electric field will be to change the kinetic energy of downwards-traveling particles. If a significant number of particles lose energy during their travel they are most likely to be deposited in their travel and therefore not to reach ground level, which is consistent with results.

For the muonic component, there is no clear consistent effect of change in response to the changes in electric and magnetic fields. This is in accordance to the fact that muons and antimuons, being more energetic, are less susceptible to the effects of change in electromagnetic field.

Further work on this subject should take into account the exact mechanisms at work in the models present in CORSIKA to create the effects observed in the results section. Additionally, it is important to state that the relation between seismic activity and changes in geoelectric and geomagnetic fields is an area in continued and constant development and further work needs to be performed in analyzing and characterizing this relation. Then, to complement this particular project it would be important to analyze if the results obtained in this study are reflected in particle detection using data from actual detectors in operation in the vicinity of seismic activity.

\section{Acknowledgements}

The authors acknowledge the financial support from USFQ. The LAGO collaboration is very grateful to all the participating institutions and The Pierre Auger collaboration for their continuous support.

\section{References}

[1] Iván Sidelnik. The Sites of the Latin American Giant Observatory. PoS, ICRC2015:665, 2016.

[2] O. Molchanov, E. Fedorov, Alexander Schekotov, E. Gordeev, V. Chebrov, V. Surkov, A. Rozhnoi, Stanislav Andreevsky, Dmitry Iudin, S. Yunga, A. Lutikov, Masashi Hayakawa, and P. Biagi. 
Lithosphere-atmosphere-ionosphere coupling as governing mechanism for preseismic short-term events in atmosphere and ionosphere. Natural Hazards and Earth System Science, 4, 112004.

[3] Marin Cavelis and Branislav Glavatovic. Possible new phenomenon in the atmosphere electric field intensity variations that is caused by the strong earthquake occurrences. 022019.

[4] M. Hayakawa, J.P. Raulin, Y. Kasahara, F. C. P. bertoni, Y. Hobara, and W. Guevara-Day. Ionospheric perturbations in possible association with the 2010 haiti earthquake, as based on medium-distance subionospheric vlf propagation data. Natural Hazards and Earth System Sciences, 11, 022011.

[5] H. Fujiwara, M. Kamogawa, M. Ikeda, J. Y. Liu, H. Sakata, Y. I. Chen, H. Ofuruton, S. Muramatsu, Y. J. Chuo, and Y. H. Ohtsuki. Atmospheric anomalies observed during earthquake occurrences. Geophysical Research Letters, 31(17), 2004.

[6] Masashi Hayakawa, Katsumi Hattori, and Kenji Ohta. Monitoring of ulf (ultra-low-frequency) geomagnetic variations associated with earthquakes. Sensors, 7(7):1108-1122, Jul 2007.

[7] Yukio Fujinawa, Kozo Takahashi, Yoichi Noda, Hiroshi Iitaka, and Shinobu Yazaki. Remote detection of the electric field change induced at the seismic wave front from the start of fault rupturing. International Journal of Geophysics, 2011, 072011.

[8] M. Parrot. Use of satellites to detect seismo-electromagnetic effects. Advances in Space Research, 15(11):27 - 35, 1995. Natural Hazards: Monitoring and Assessment Using Remote Sensing Technique.

[9] Enrique G. Cordaro, Patricio Venegas, and David Laroze. Latitudinal variation rate of geomagnetic cutoff rigidity inthe active chilean convergent margin. Annales Geophysicae, 36, 032018.

[10] Abhijit Choudhury, Anirban Guha, Barin De, and Rakesh Roy. A statistical study on precursory effects of earthquakes observed through the atmospheric vertical electric field in northeast india. Annals of Geophysics, 56(3):0331, 2013.

[11] D. Heck, J. Knapp, J.N. Capdevielle, G. Schatz, and T. Thouw. CORSIKA: A Monte Carlo code to simulate extensive air showers. 21998.

[12] A.J. Rubio-Montero, R. Pagán-Muñoz, R. Mayo-García, A. Pardo-Diaz, I. Sidelnik, H. Asorey, and the LAGO Collaboration. The EOSC-Synergy cloud services implementation for the Latin American Giant Observatory (LAGO). In 37th ICRC, number 261, pages 1-4, Berlin, Germany, 2021.

[13] Christian Sarmiento-Cano, Mauricio Suárez-Durán, Rolando Calderón-Ardila, Adriana VásquezRamírez, Andrei Jaimes-Motta, Sergio Dasso, Iván Sidelnik, Luis A. Núñez, and Hernán Asorey. Performance of the LAGO water Cherenkov detectors to cosmic ray flux. 102020.

[14] National Geophysical Data Center. Neci geomagnetic calculatot. https://www.ngdc.noaa.gov/ geomag/calculators/magcalc. shtml\#igr fwmm. Accessed: 2020-11-14. 


\section{Full Authors List: LAGO Collaboration}

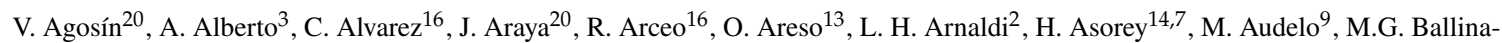
Escobar $^{19}$, D. C. Becerra-Villamizar ${ }^{18}$, X. Bertou ${ }^{2}$, K.S. Caballero-Mora ${ }^{16}$, R. Caiza ${ }^{8}$, R. Calderón-Ardila ${ }^{14}$, Calle, J. ${ }^{24}$, A. C. Fauth ${ }^{27}$, E. Carrera Jarrin ${ }^{26}$, L. E. Castillo Delacroix ${ }^{11}$, C. Castromonte ${ }^{25}$, Cazar-Ramírez D. ${ }^{26}$, Diego Cogollo ${ }^{28}$, D. A. Coloma Borja ${ }^{26}$, R. Conde ${ }^{1}$, J. Cotzomi ${ }^{1}$, D. Dallara ${ }^{11}$, S. Dasso ${ }^{13,5,6}$, R. Aguiar27 ${ }^{27}$, Albuquerque, A. ${ }^{28}$, J.H.A.P.Reis ${ }^{27}$, H. De León ${ }^{16}$, R. deLeónBarrios $^{23}$, D. Domínguez ${ }^{8}$, M. Echiburu ${ }^{21}$, M. González², M. Gómez Berisso ${ }^{2}$, J. Grisales Casadiegos ${ }^{23}$, A. M. Gulisano ${ }^{13,12,6}$, Juan Carlos Helo ${ }^{17}$, Condori, C. A. H. ${ }^{24}$, J. E. Ise ${ }^{11}$, Nascimento, G. K. M ${ }^{28}$, M. A. Leigui de Oliveira ${ }^{29}$, F. L. Miletto ${ }^{27}$, V. P. Luzio ${ }^{29}$, F. Machado $^{25}$, Juan F. Mancilla-Caceres ${ }^{22}$, D. Manriquez ${ }^{20}$, A. Martínez-Méndez ${ }^{23}$, O. Martinez ${ }^{1}$, R. Mayo-García ${ }^{3}$, L.G. Mijangos ${ }^{22}$, Miranda, $\mathrm{P}^{24}$, M. G. Molina ${ }^{11}$, I.R. Morales ${ }^{19}$, O.G Morales-Olivares ${ }^{16}$, E. Moreno-Barbosa ${ }^{1}$, P. Muñoz ${ }^{17}$, Nina, $\mathrm{C}^{24}$, L.A. Núñez ${ }^{23}$, L. Otininano ${ }^{4}$, R. Pagán-Muñoz ${ }^{3}$, K. M. Parada-Jaime ${ }^{18}$, H. M. Parada-Villamizar ${ }^{18}$, R. Parra ${ }^{10}$, J. Peña-Rodríguez ${ }^{23}$, M. Pereira ${ }^{13}$, Y. A. Perez-Cuevas ${ }^{18}$, H. Perez ${ }^{19}$, J. Pisco-Guabave ${ }^{23}$, Raljevic, M. $^{24}$, M. Ramelli ${ }^{13}$, C. Ramírez ${ }^{22}$, Rivera, $\mathrm{H}^{24}$, L. T. Rubinstein ${ }^{13}$, A.J. Rubio-Montero $^{3}$, J.R. Sacahui ${ }^{19}$, H. Salazar ${ }^{1}$, N. Salomón ${ }^{11}$, J. Samanes ${ }^{4}$, N.A. Santos ${ }^{5}$, C. Sarmiento-Cano ${ }^{14}$, I. Sidelnik ${ }^{2}$, Mayra B. Silva $^{22}$, O. Soto ${ }^{17}$, M. Suárez-Durán ${ }^{18,31}$, Subieta Vasquez, M. ${ }^{24}$, Terrazas C. ${ }^{24}$, Ticona, R. ${ }^{24}$, T. Torres Peralta ${ }^{11}$, Pablo A. Ulloa ${ }^{17}$, Z.R. Urrutia ${ }^{22}$, N. Vásquez ${ }^{8}$, A. Vázquez-Ramírez ${ }^{23}$, A. Vega ${ }^{20}$, P. Vega $^{17}$, J. Vega $^{4}$, A. Vesga-Ramirez ${ }^{14}$, D. Vitoreti ${ }^{30}$, R. Wiklich Sobrinho ${ }^{29}$,

${ }^{1}$ Benemérita Universidad Autónoma de Puebla". ${ }^{2}$ Centro Atómico Bariloche (CNEA/CONICET/IB)". ${ }^{3}$ CIEMAT". ${ }^{4}$ Comisión Nacional de Investigación y Desarrollo Aeroespacial". ${ }^{5}$ Departamento de Ciencias de la Atmósfera y los Océanos, Facultad de Ciencias Exactas y Naturales, Universidad de Buenos Aires.". ${ }^{6}$ Departamento de Física (FCEN,UBA)". ${ }^{7}$ Departamento Física Médica, CNEA-CONICET-UNSAM". ${ }^{8}$ Escuela Politécnica Nacional". ${ }^{9}$ Escuela Superior Politécnica de Chimborazo". ${ }^{10}$ European Soutern Observatory (ESO)". ${ }^{11}$ Facultad de Ciencias Exactas y Tecnología (FACET) - Universidad Nacional de Tucumán (UNT)". ${ }^{12}$ Instituto Antártico Argentino, Dirección Nacional del Antartico, Instituto de Astronomía y Física del Espacio (UBA-CONICET)". ${ }^{13}$ Instituto de Astronomía y Física del Espacio, IAFE (UBA-CONICET)". ${ }^{14}$ Instituto de Tecnologías en Detección y Astropartículas (CNEA, CONICET,UNSAM)". ${ }^{16}$ Universidad Autónoma de Chiapas". ${ }^{17}$ Universidad de La Serena". ${ }^{18}$ Universidad de Pamplona". ${ }^{19}$ Universidad de San Carlos". ${ }^{20}$ Universidad de Valparaíso". ${ }^{21}$ Universidad de Viña del Mar". ${ }^{22}$ Universidad del Valle de Guatemala". ${ }^{23}$ Universidad Industrial de Santander". ${ }^{24}$ Universidad Mayor de San Andrés". ${ }^{25}$ Universidad Nacional de Ingeniería". ${ }^{26}$ Universidad San Francisco de Quito". ${ }^{27}$ Universidade Estadual de Campinas". ${ }^{28}$ Universidade Federal de Campina Grande". ${ }^{29}$ Universidade Federal do ABC". ${ }^{30}$ Universidade Federal do Recôncavo da Bahia". ${ }^{31}$ Université Libre de Bruxelles, Brussels, Belgium.". 